\title{
The Effect of Enhanced Depression Care on Adherence to Risk Reducing Behaviors after Acute Coronary Syndromes: Findings from the COPES Trial
}

\author{
Ian M. Kronish, MD, MPH ${ }^{1}$, Nina Rieckmann, $\mathrm{PhD}^{2}$, Matthew M. Burg, $\mathrm{PhD}^{1,3}$, Donald \\ Edmondson, $\mathbf{P h D}^{1}$, Joseph E. Schwartz, $\mathbf{P h D}^{1,4}$, and Karina W. Davidson, $\mathbf{P h D}^{1}$ \\ ${ }^{1}$ Center for Behavioral Cardiovascular Health, Columbia University Medical Center, New York, \\ NY \\ ${ }^{2}$ Berlin School of Public Health, Charité Universitäts medizin, Berlin, Germany \\ ${ }^{3}$ Section of Cardiovascular Medicine, Yale University School of Medicine, New Haven, CT \\ ${ }^{4}$ Applied Behavioral Medicine Research Institute, Stony Brook University, Stony Brook, NY
}

\section{Abstract}

\begin{abstract}
Background-The presence of depression after an acute coronary syndrome (ACS) is associated with worse prognosis and lower adherence to risk reducing behaviors. We reported earlier that an enhanced depression care intervention reduces depression symptoms and major adverse cardiac events. This study evaluates the impact of the depression intervention on health behavior and blood pressure control.
\end{abstract}

Methods-Between 2005 and 2008, 157 patients who remained persistently depressed 3-months after ACS were randomized to a 6 month depression intervention (initial patient preference for problem-solving therapy and/or pharmacotherapy, followed by stepped-care; 80 patients) or to usual care (77 patients). At randomization, and then 2, 4, and 6 months later, patients were asked if they 1) missed taking their aspirin; 2) followed a heart healthy diet; 3) exercised regularly; and 4) used tobacco products. Blood pressure was measured prior to randomization and 6 months later.

Results-At the end of the intervention, there was no significant improvement in the percentage of intervention compared to control group patients that adhered to aspirin ( $+3 \%$ versus $-1 \%$, $\mathrm{p}=0.23)$; followed a healthy diet $(+10 \%$ versus $+8 \%, \mathrm{p}=0.39)$; exercised regularly $(+5 \%$ versus $+4 \%, \mathrm{p}=0.65)$; abstained from tobacco $(-3 \%$ versus $-1 \%, \mathrm{p}=0.77)$; or had controlled blood pressure $(+6 \%$ versus $+16 \%, \mathrm{p}=0.26)$.

Conclusion-Despite improving depression, enhanced depression care after an ACS did not improve health behavior or blood pressure control compared to usual care. Research is needed to test whether adding an adherence intervention to enhanced depression care can improve adherence and cardiovascular prognosis in depressed post-ACS patients.

(C) 2012 Mosby, Inc. All rights reserved.

Corresponding author: Ian M Kronish, MD, MPH Center for Behavioral Cardiovascular Health, Columbia University Medical Center, PH9-311, 622 W168th Street, New York, NY 10032; ik2293@ columbia.edu; phone: 212-342-1335.

Disclosures: None

Publisher's Disclaimer: This is a PDF file of an unedited manuscript that has been accepted for publication. As a service to our customers we are providing this early version of the manuscript. The manuscript will undergo copyediting, typesetting, and review of the resulting proof before it is published in its final citable form. Please note that during the production process errors may be discovered which could affect the content, and all legal disclaimers that apply to the journal pertain. 


\section{Introduction}

The presence of depression after an acute coronary syndrome(myocardial infarction, unstable angina; ACS) is an independent risk factor for recurrent cardiovascular events and mortality. Depression after an ACS is also associated with lower adherence to cardiovascular risk reducing behaviors such as physical activity, smoking cessation, and taking cardiovascular medications. ${ }^{3-6}$ Lower adherence to these healthy behaviors may explain a portion of the association between depression and worse prognosis in patients with either stable coronary heart disease (CHD)or after ACS. ${ }^{7-9}$ Despite the strong association between depression and non-adherence in observation studies, it remains unknown whether treatment of depression improves patients' adherence to healthy behaviors after ACS.

In the Coronary Psychosocial Evaluation Studies (COPES)randomized controlled trial, we previously found that an enhanced depression care intervention comprised of patient preference for problem-solving therapy (PST), antidepressant medications, or both, followed by stepped care according to treatment response, was effective at reducing depressive symptoms after an ACS. ${ }^{10}$ At the end of the trial, patients in the intervention group were more likely to be taking antidepressant medications and participating in psychotherapy. ${ }^{10}$ Moreover, they had a $31 \%$ reduction in their depressive symptoms, while participants in the usual care group only had a $9 \%$ reduction in depressive symptoms. With a standardized effect size of 0.59 , this intervention compares favorably with other evidence-based interventions for depression in patients with cardiovascular disease. ${ }^{11}$ Patients randomized to this intervention also had a lower occurrence of major adverse coronary events(MACE) during the intervention period. ${ }^{10}$

Because we had found a reduced MACE rate in the intervention group, we tested in this secondary analysis the impact of enhanced depression care on self-reported risk reducing behaviors including regularly taking prescribed medications, following a heart healthy diet, exercising regularly, and abstaining from tobacco products. We also assessed the impact of the intervention on objectively measured blood pressure control, a cardiac risk factor that is partially mediated by adherence to exercise, diet, and medications. We hypothesized that as compared to usual care, enhanced depression care would result in better adherence to risk reducing behaviors after an ACS and better blood pressure control, and that this improvement might explain in part the improved MACE rate in the COPES depression trial.

\section{Methods}

The COPES trial design is described elsewhere. ${ }^{12}$ Briefly, 157 hospitalized ACS patients who were persistently depressed (Beck Depression Inventory (BDI) ${ }^{13}$ score $\geq 10$ within 1week of hospitalization and again 3 months later) were recruited from 5 US hospitals(Mount Sinai Hospital and New York Presbyterian Hospital, New York, New York; and New Haven Hospital, Hospital of St Raphael, and Veterans Affairs Connecticut Healthcare System-West Haven, New Haven, Connecticut) between 2005 and 2008 and randomized to receive six months of enhanced depression care or to usual care. Exclusion criteria were alcohol or drug dependency, dementia, psychosis, bipolar disorder, terminal illness, unavailability for follow-up, BDI score of $\geq 45$, or suicidality. The institutional review boards at all institutions approved the protocol, and all participants provided written informed consent. This work was supported by grants HC-25197, HL-088117, HL-101663, HL-84034 from the National Heart, Lung, and Blood Institute and by Columbia University's CTSA grant UL1RR024156 from NCATS-NCRR/NIH. In addition, Dr. Kronish was supported by grant K23HL098359 from the National Heart, Lung, and Blood Institute and Dr. Edmondson was supported by grant KM1-CA156709 from the National Cancer Institute. The authors are 
solely responsible for the design and conduct of this study, all study analyses and drafting and editing of the paper.

Enhanced depression care consisted of patient preference for either brief problem solving therapy (PST) and/or pharmacotherapy, followed by stepped-care in which symptom severity was reviewed every 6 to 8 weeks and treatment was augmented, maintained, or switched according to predetermined decision rules. PST therapists did not assess or advise patients on adherence issues unless the patient chose this issue as a focus for their PST sessions. The risk of contamination between groups was minimized as enhanced depression care visits took place in research settings rather than at clinical sites. Usual care consisted of notifying treating physician(s) about their patients' depressive status; patients can then receive whichever care for depressive symptoms that their clinician(s) feels is warranted.

\section{Measures}

Adherence was assessed via patient interview by assessors who were blinded to patients' group assignment. Specifically, at the time of ACS, patients were asked to report "Yes/No" whether, in the 3 months prior, 1) they were exercising regularly; 2) they were following a low fat, low cholesterol diet; and 3) whether in the prior week they had used tobacco products. A trandomization (3 months after the index ACS) and 2, 4, and 6 months later, adherence to these behaviors since the prior study visit was assessed using the same questions. In addition, patients were asked if they had missed taking their aspirin on any day since that prior visit. Similar questions were used to assess adherence after ACS assessments 2 and 4 months after randomization were made over the telephone; all other assessments were done in-person.

Blood pressure was recorded at 3 in-person study visits (ACS hospitalization, 3 months and 9 months later)as the average of two measurements from the same non-dominant arm using an appropriately sized cuff and a mercury sphygmomanometer. Measurements were taken at least two minutes apart and after the patient had been seated for at least 5 minutes. Blood pressure was measured at the pre-randomization visit (3 months after hospitalization) and 6 months later. Blood pressure control was defined as having a systolic blood pressure less than $140 \mathrm{mmHg}$ and a diastolic blood pressure less than $90 \mathrm{mmHg}$.

Other variables including patient demographics, alcohol use, and cardiac rehabilitation participation were assessed through patient interview. At-risk alcohol use was defined as more than two drinks per day for men and more than one drink per day for women. Medical characteristics including left ventricular ejection fraction and cardiac disease prognosis as measured by the Global Registry of Acute Coronary Events ${ }^{14}$ score were assessed through chart abstraction.

\section{Statistical Analysis}

Of the 80 intervention patients and the 77 usual care patients, $81 \%$ and $92 \%$ had complete data on self-reported adherence and $79 \%$ and $85 \%$ had complete data on blood pressure, respectively. Chi-square tests revealed that patients who reported being non-adherent at the time of the index hospitalization were not more likely to have subsequent missing data. Missing data were handled using multiple imputation (five datasets); multiple imputation was performed separately for each adherence behavior. Variables that were associated with adherence to health behaviors in prior studies (age, gender, race, ethnicity, depression severity) were used as predictors in the multiple imputation procedure. ${ }^{3}$

In an exploratory analysis, we used McNemar's test to assess whether adherence to risk reducing behaviors significantly improved between the index ACS hospitalization and the pre-randomization visit 3 months later, irrespective of group assignment. For our main 
analysis, we used logistic regression, pooling the results across the five multiply imputed datasets, to assess whether the intervention was associated with increased odds of adherence to aspirin, diet, exercise, and abstinence from smoking, as well as blood pressure control during the intervention period. In each analysis, adherence at the beginning of the intervention period was treated as a covariate. For blood pressure, we also used t-tests to compare the mean blood pressure at 9 months and the change in blood pressure between 3 months and 9 months in enhanced versus usual care group patients. We also estimated the sample sizes that would have been needed to detect significant effects of the COPES intervention on adherence given the odds ratios found in these analyses and assuming $80 \%$ power. All analyses were performed using SPSS statistical software (version 18, SPSS Inc., Chicago, IL).

\section{Results}

As has been previously reported, the mean age of patients in the trial was 60 years, $54 \%$ were women, $43 \%$ were Hispanic, and $17 \%$ were Black. ${ }^{10}$ Enhanced and usual care patients were similar on demographic and medical characteristics at the time of the index ACS hospitalization(Table 1).

At the time of the index hospitalization, there were no significant differences in the percentage of patients in each group who reported exercising regularly, following a heart healthy diet, never missing their medications, and abstaining from tobacco (Figure). Other than for at-risk alcohol use, adherence to healthy behaviors was suboptimal 3 months after the index ACS hospitalization (the time of randomization), and ranged from a low of $43 \%$ of participants regularly exercising to a high of $83 \%$ of participants abstaining from tobacco (Figure).No patients in either group reported at-risk alcohol use at 3 months and only 1 patient (in the control group) reported at-risk alcohol use at 9 months. The percentage of patients participating in cardiac rehabilitation at 3 months was similar in both groups (22\% intervention versus $16 \%$ usual care; $\mathrm{p}=0.36$ ).

Irrespective of randomization assignment, there was a significant $(\mathrm{p}<0.05)$ increase in the percentage of patients who reported being adherent to following a healthy diet (45\% to $66 \%$ )and to regular exercise (29\% to 43\%), 3 months after the index ACS hospitalization versus at the time of the index ACS. In contrast, there was no significant change in the percentages of the intervention and usual care groups that abstained from smoking ( $81 \%$ to $83 \%$ ) or had controlled blood pressure ( $78 \%$ to $69 \%$ ) over this time period, respectively. Similarly, there were no significant differences in blood pressure at 9 months or in the change in systolic blood pressure between 9 months and 3 months.

During the intervention period, there were small relative improvements in adherence to aspirin, healthy diet, and regular exercise, but these improvements were not statistically significant (Table 2; Figure). Furthermore, there were no significant differences in mean systolic blood pressure at 9 months, nor in the change in systolic blood pressure between 3 months and 9 months between both groups (data not shown). We estimated that we would have needed to enroll from 872 patients in the case of aspirin adherence to 6039 patients in the case of adherence to regular exercise to have sufficient sample size to find statistically significant differences in these health behaviors with the odds ratios found in our study. The effect of the intervention on use of tobacco products and blood pressure control were in the direction of causing harm, but again, these differences were not statistically significant. The results showing no difference were the same when analyzed using list wise deletion and when performed with the assumption that missing data represented non-adherence (data not shown). 


\section{Discussion}

Despite resulting in improved depressive symptoms, increased receipt of psychotherapy and antidepressants, and a lower MACE rate, the COPES enhanced depression care intervention did not have a comparable beneficial effect on self-reported adherence to cardiovascular risk reducing behaviors including taking aspirin every day, following a healthy diet, regularly exercising, and abstaining from tobacco products. Similarly, the COPES intervention did not improve the proportion of patients with controlled blood pressure. A number of biological mechanisms such as platelet hyperreactivity and autonomic dysfunction have been proposed to explain the association between depression and poor post-ACS prognosis. Hence, it is possible that enhanced depression care may improve post-ACS outcomes by acting on biological pathways not measured in this study. For example, patients with greater declines in depressive symptoms as a result of enhanced depression care may have had less reactive platelets and greater heart rate variability which, in turn, could have reduced the risk of recurrent MI and sudden cardiac death, respectively.

Although our finding that enhanced depression care did not improve adherence was contrary to the hypothesis generated by observational studies, our finding was consistent with the results of a prior trials of enhanced depression care incardiac ${ }^{17}$ and diabetic patients ${ }^{18}$ with comorbid depression. In both cases, despite improving depressive symptoms, enhanced depression care did not improve self-reported adherence by the end of the trial. Our study expands upon this literature by testing the effect of a more intensive depression intervention targeted at patients with persistently elevated depressive symptoms after ACS. Furthermore, our study assesses the effect of the intervention on smoking and blood pressure, key cardiovascular risk factors. Thus, enhanced depression care, alone, may not be adequate for improving adherence to health behaviors related to comorbid medical illness.

There are several additional potential interpretations of the current findings. First, it is possible that treating depression may be necessary but not sufficient to improve health behavior in patients with comorbid medical illness. For example, Trockel and colleagues reported that enhanced depression care resulted in increased smoking cessation in post-MI patients only for patients with adequate social support. ${ }^{19}$ Another possibility is that despite the relatively strong effect size of the COPES intervention on depressive symptoms, this and other effective depression interventions may not be potent enough to produce the increased energy, motivation, and concentration that may be necessary to have a significant impact on adherence behaviors.

Although the COPES intervention did not improve health behaviors, we observed significant improvements in adherence to exercise and healthy diet three months after hospitalization for an ACS (prior to the start of the intervention) in the sample as a whole. This substantiates the potential for cardiovascular events to serve as the basis for a "teachable moment" that can spur behavior change even among the persistently depressed patients who were eligible for this study. ${ }^{20}$

There are several limitations to our conclusion that enhanced depression care does not result in improved adherence. First, these were post-hoc analyses. ${ }^{21}$ Of greater concern, adherence behaviors were measured using single-item, dichotomous, self-report questions and thus were imprecise. In addition, self-report can inflate the true rate of adherence due to recall or social desirability bias. ${ }^{22}$ Nevertheless, in a prior observational study, we found that changes in responses to similar questions for diet, exercise, and smoking were associated with changes in depression in the first 3 months after ACS. ${ }^{13}$ In addition, we were reassured that the questions assessing diet and exercise were sensitive to change between hospitalization and 3 months in the current study. Future studies should consider carefully measuring 
adherence using either validated scales or objective instruments such as electronic monitors for medication adherence and actigraphs for exercise. In addition, it is possible that enhanced depression care could have a delayed effect on adherence behaviors after the conclusion of the trial as has been found in one prior trial of enhanced depression care in cardiac patients, ${ }^{17}$ but we did not test for this in our study. Finally, this study may have been underpowered for these secondary analyses, though even if the enhanced depression intervention did improve adherence to some behaviors, these improvements were unlikely to be clinically meaningful.

\section{Implications}

Rates of adherence to risk reducing behaviors were suboptimal for both enhanced depression and usual care patients and thus represent appropriate targets for intervention. Future trials of enhanced depression care that seek to improve cardiovascular outcomes along with depression symptoms might consider multi-modal approaches that incorporate depression treatment with adherence training. ${ }^{23}$ A collaborative care depression intervention in patients with diabetes or stable coronary heart disease that targeted not only depression, but also cardiovascular risk factor control via more aggressive medication intensification and adherence counseling resulted in improvements in depression as well as diabetes, cholesterol, and blood pressure control, but not in adherence to health behaviors. Hence, future trials will need to consider alternative approaches to improving health behavior in depressed cardiovascular patients. Determining whether to offer depression treatment prior to intervening on adherence behaviors or whether to address depression and non-adherence simultaneously is another important consideration when designing future interventions for depressed post-ACS patients. ${ }^{26}$

\section{Acknowledgments}

None

Funding Sources: This work was supported by grants HC-25197, HL-088117, HL-101663, HL-84034 from the National Heart, Lung, and Blood Institute and by Columbia University's CTSA grant UL1-RR024156 from NCATS-NCRR/NIH. Dr. Kronish is supported by grant K23-HL098359 from the National Heart, Lung, and Blood Institute. Dr. Edmondson is supported by grant KM1-CA156709 from the National Cancer Institute. The content is solely the responsibility of the authors and does not necessarily represent the official views of the NIH.

\section{References}

1. Nicholson A, Kuper H, Hemingway H. Depression as an aetiologic and prognostic factor in coronary heart disease: a meta-analysis of 6362 events among 146-538 participants in 54 observational studies. Eur Heart J. 2006; 27:2763-74. [PubMed: 17082208]

2. van Melle JP, de Jonge P, Spijkerman TA, et al. Prognostic association of depression following myocardial infarction with mortality and cardiovascular events: a meta-analysis. Psychosomatic Medicine. 2004; 66:814-22. [PubMed: 15564344]

3. Kronish IM, Rieckmann N, Halm EA, et al. Persistent depression affects adherence to secondary prevention behaviors after acute coronary syndromes. J Gen Intern Med. 2006; 21:1178-83. [PubMed: 16899061]

4. Rieckmann N, Gerin W, Kronish IM, et al. Course of depressive symptoms and medication adherence after acute coronary syndromes: an electronic medication monitoring study. J Am Coll Cardiol. 2006; 48:2218-22. [PubMed: 17161249]

5. Ziegelstein RC, Fauerbach JA, Stevens SS, Romanelli J, Richter DP, Bush DE. Patients with depression are less likely to follow recommendations to reduce cardiac risk during recovery from a myocardial infarction. Arch Intern Med. 2000; 160:1818-23. [PubMed: 10871976] 
6. Bauer LK, Caro MA, Beach SR, et al. Effects of depression and anxiety improvement on adherence to medication and health behaviors in recently hospitalized cardiac patients. Am J Cardiol. 2012; 109:1266-71. [PubMed: 22325974]

7. Carney RM, Freedland KE, Miller GE, Jaffe AS. Depression as a risk factor for cardiac mortality and morbidity: a review of potential mechanisms. J Psychosom Res. 2002; 53:897-902. [PubMed: 12377300]

8. Whooley MA, de Jonge P, Vittinghoff E, et al. Depressive symptoms, health behaviors, and risk of cardiovascular events in patients with coronary heart disease. JAMA. 2008; 300:2379-88. [PubMed: 19033588]

9. Rieckmann N, Burg MM, Kronish IM, Chaplin WF, Schwartz JE, Davidson KW. Aspirin Adherence, Depression and One-Year Prognosis after Acute Coronary Syndrome. Psychother Psychosom. 2011; 80:316-8. [PubMed: 21720196]

10. Davidson KW, Rieckmann N, Clemow L, et al. Enhanced depression care for patients with acute coronary syndrome and persistent depressive symptoms: coronary psychosocial evaluation studies randomized controlled trial. Arch Intern Med. 2010; 170:600-8. [PubMed: 20386003]

11. Baumeister H, Hutter N, Bengel J. Psychological and pharmacological interventions for depression in patients with coronary artery disease. Cochrane database of systematic reviews (Online). 2011; 9:CD008012. [PubMed: 21901717]

12. Burg MM, Lesperance F, Rieckmann N, Clemow L, Skotzko C, Davidson KW. Treating persistent depressive symptoms in post-ACS patients: The project COPES phase-I randomized controlled trial. Contemp Clin Trials. 2007

13. Beck AT, Ward CH, Mendelson M. An inventory for measuring depression. Archives of General Psychiatry. 1961; 4:561-71. [PubMed: 13688369]

14. Eagle KA, Lim MJ, Dabbous OH, et al. A validated prediction model for all forms of acute coronary syndrome: estimating the risk of 6-month postdischarge death in an international registry. JAMA. 2004; 291:2727-33. [PubMed: 15187054]

15. Lett HS, Blumenthal JA, Babyak MA, et al. Depression as a risk factor for coronary artery disease: evidence, mechanisms, and treatment. Psychosom Med. 2004; 66:305-15. [PubMed: 15184688]

16. Rieckmann N, Kronish IM, Haas D, et al. Persistent depressive symptoms lower aspirin adherence after acute coronary syndromes. Am Heart J. 2006; 152:922-7. [PubMed: 17070160]

17. Huffman JC, Mastromauro CA, Sowden G, Fricchione GL, Healy BC, Januzzi JL. Impact of a depression care management program for hospitalized cardiac patients. Circ Cardiovasc Qual Outcomes. 2011; 4:198-205. [PubMed: 21386067]

18. Lin EH, Katon W, Rutter C, et al. Effects of enhanced depression treatment on diabetes self-care. Ann Fam Med. 2006; 4:46-53. [PubMed: 16449396]

19. Trockel M, Burg M, Jaffe A, Barbour K, Taylor CB. Smoking behavior postmyocardial infarction among ENRICHD trial participants: cognitive behavior therapy intervention for depression and low perceived social support compared with care as usual. Psychosom Med. 2008; 70:875-82. [PubMed: 18842753]

20. Cohen DJ, Clark EC, Lawson PJ, Casucci BA, Flocke SA. Identifying teachable moments for health behavior counseling in primary care. Patient Educ Couns. 2010

21. Marler JR. Secondary analysis of clinical trials--a cautionary note. Prog Cardiovasc Dis. 2012; 54:335-7. [PubMed: 22226000]

22. Shi L, Liu J, Koleva Y, Fonseca V, Kalsekar A, Pawaskar M. Concordance of adherence measurement using self-reported adherence questionnaires and medication monitoring devices. Pharmacoeconomics. 2010; 28:1097-107. [PubMed: 21080735]

23. Antoni MH, Carrico AW, Duran RE, et al. Randomized clinical trial of cognitive behavioral stress management on human immunodeficiency virus viral load in gay men treated with highly active antiretroviral therapy. Psychosom Med. 2006; 68:143-51. [PubMed: 16449425]

24. Katon W, Lin EH, Von Korff M, et al. Integrating depression and chronic disease care among patients with diabetes and/or coronary heart disease: the design of the TEAMcare study. Contemp Clin Trials. 2010; 31:312-22. [PubMed: 20350619]

25. Katon WJ, Lin EH, Von Korff M, et al. Collaborative care for patients with depression and chronic illnesses. N Engl J Med. 2010; 363:2611-20. [PubMed: 21190455] 
26. Hyman DJ, Pavlik VN, Taylor WC, Goodrick GK, Moye L. Simultaneous vs sequential counseling for multiple behavior change. Arch Intern Med. 2007; 167:1152-8. [PubMed: 17563023] 

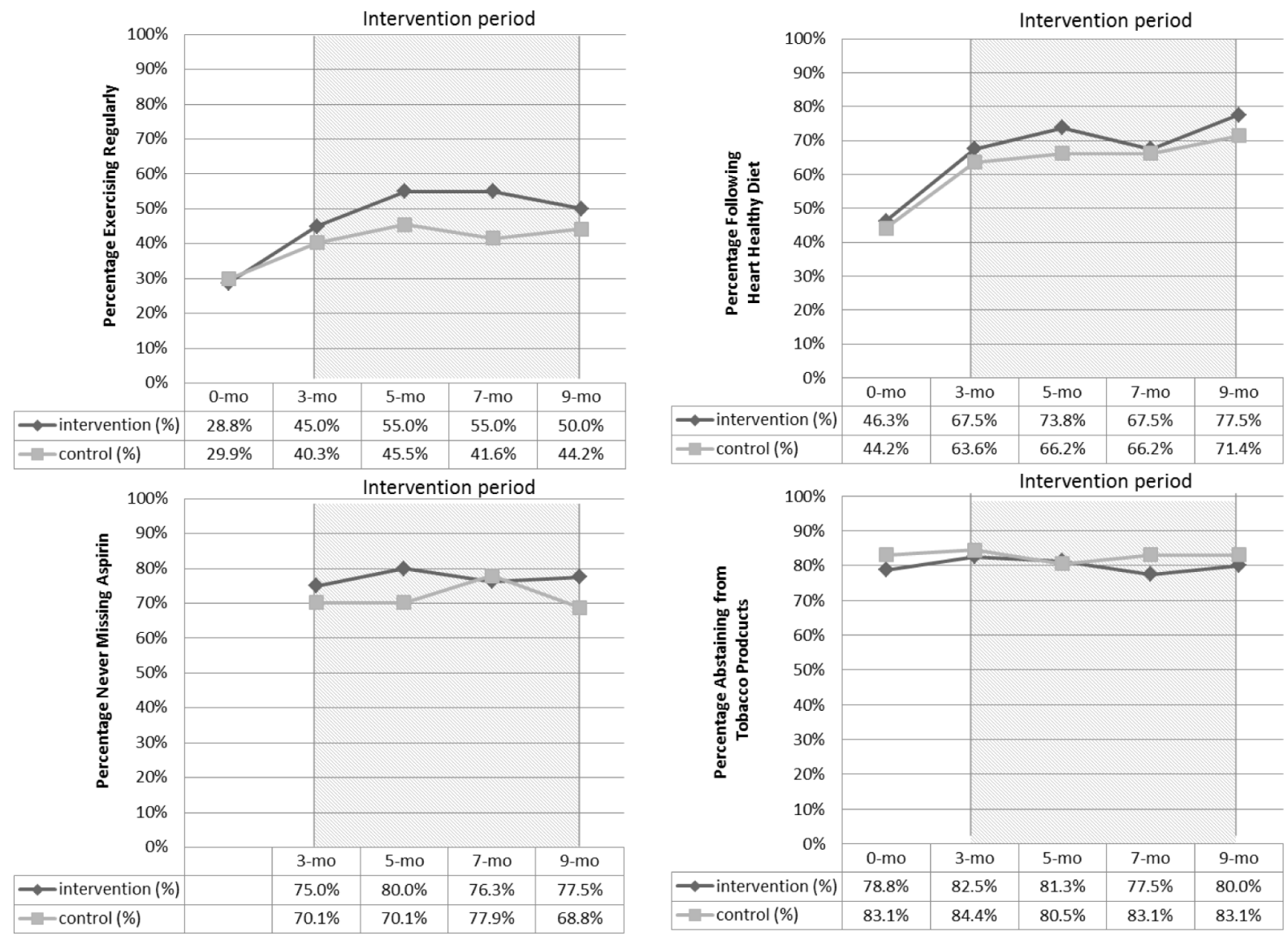

Figure 1.

Percentage of Patients in Intervention and Usual Care Groups Who Adhere to Risk

Reducing Behaviors after Acute Coronary Syndromes.* Top Left: Never Missing Aspirin;

Top Right: Following Heart Healthy Diet; Bottom Left: Exercising Regularly; Bottom

Right: Abstaining from Tobacco Products

*Randomization occurred at 3 months 
Table 1

Patient Characteristics at Prior to Randomization into the COPES Enhanced Depression Care Randomized Controlled Trial ${ }^{*}$

\begin{tabular}{|c|c|c|c|}
\hline Characteristic & Intervention $(\mathrm{n}=\mathbf{8 0})$ & Usual Care $(n=77)$ & P-Value \\
\hline Age, mean in years (SD) & $59.3(10.6)$ & $61.1(10.6)$ & 0.29 \\
\hline Female & $43(54)$ & $41(53)$ & 0.95 \\
\hline Black & $12(15)$ & $17(22)$ & 0.25 \\
\hline Hispanic & $36(45)$ & $32(42)$ & 0.66 \\
\hline \multicolumn{4}{|c|}{ Type of acute coronary syndrome } \\
\hline Myocardial infarction & $21(26)$ & $17(22)$ & 0.69 \\
\hline Unstable angina & $58(73)$ & $60(78)$ & \\
\hline GRACE score, mean (SD) & $91(24)$ & $94(23)$ & 0.48 \\
\hline LVEF $<40 \%$ & $7(11)$ & $7(11)$ & 0.99 \\
\hline
\end{tabular}

Unless otherwise indicated, data are expressed as number (percentage) of patients.

Abbreviations: SD, standard deviation; GRACE, Global Registry of Acute Coronary Events;LVEF, left ventricular ejection fraction 


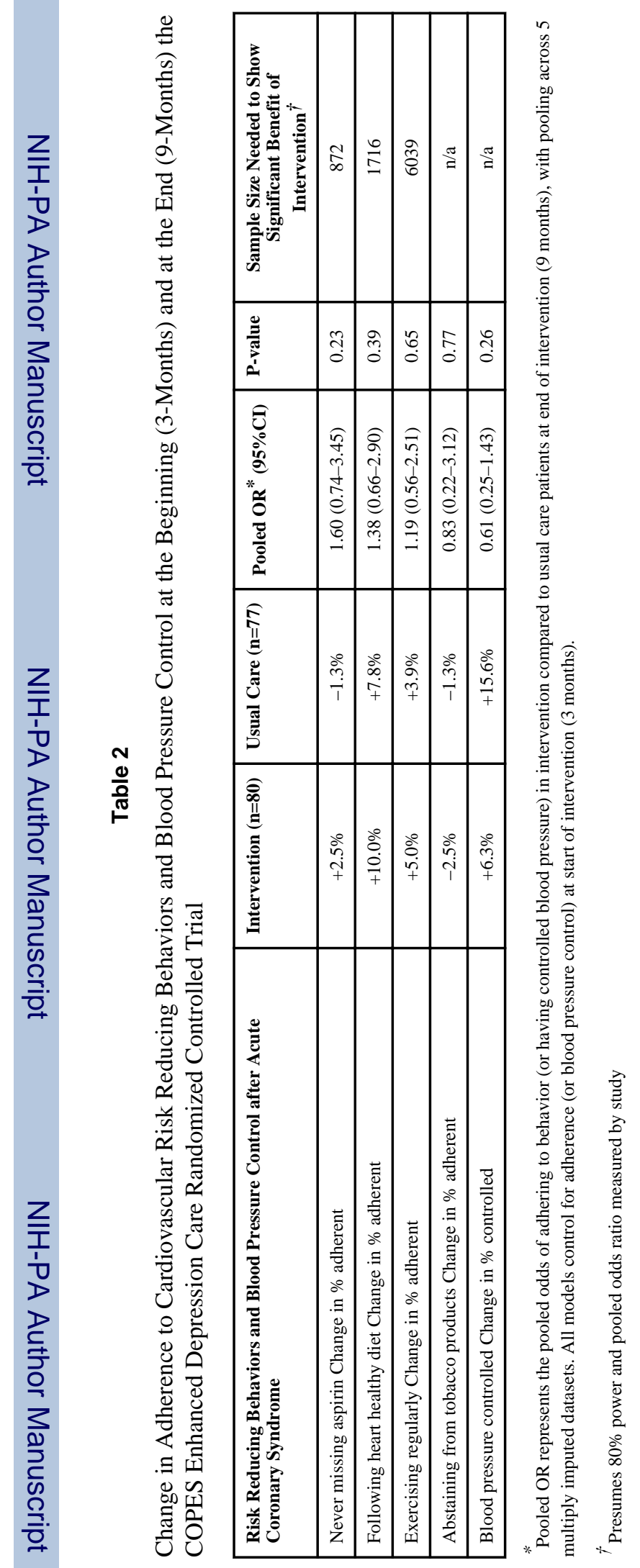

Am Heart J. Author manuscript; available in PMC 2013 October 01. 